\title{
Potassium restores vasorelaxation of resistance arterioles in non-hypertensive DOCA/salt fed mice
}

\author{
C. Wyss ${ }^{\text {a, } 1}$, Q. Wang b,e,f,g,1 ${ }^{\text {, D. Golshayan }}{ }^{\text {b }}$, J. Nussberger ${ }^{\text {c }}$, M. Burnier ${ }^{\text {b }}$, H.A. Lehr ${ }^{\text {a,* }}{ }^{*}$ S.C. Schaefer ${ }^{\text {d }}$ \\ a Institute of Pathology, Centre Hospitalier Universitaire Vaudois, Lausanne, Switzerland \\ b Service of Nephrology, Centre Hospitalier Universitaire Vaudois, Lausanne, Switzerland \\ c Service of Angiology, Centre Hospitalier Universitaire Vaudois, Lausanne, Switzerland \\ d Institute of Pathology, Inselspital, Bern, Switzerland \\ e Division of Physiology, Department of Medicine, University of Fribourg, Switzerland \\ ${ }^{\mathrm{f}}$ Chinese Hypertension League Institute, Beijing, China \\ ${ }^{g}$ Huazhong University of Science and Technology, Wuhan, China
}

\begin{abstract}
Background: Potassium-enriched diets exert renal and cardiovascular protective effects, but the underlying mechanisms are largely unknown.

Methods: Using the dorsal skinfold chamber model for intravital microscopy, we examined endotheliumdependent vasorelaxation of precapillary resistance arterioles in response to acetylcholine or the NO donor SNAP in awake mice. Experiments were performed in uni-nephrectomized one renin gene (Ren-1c) C57BL/6 mice (control group) and in mice having received a continuous administration of deoxycorticosterone acetate and a dietary supplementation of $1 \%$ sodium chloride for 8 weeks (DOCA/salt group). An additional group of DOCA/salt treated animals received a dietary supplement of $0.4 \% \mathrm{KCl}$ for 3 weeks prior to the experiments (DOCA/salt + potassium group).

Results: DOCA/salt treatment for 8 weeks resulted in hypokalemia, but blood pressure remained unchanged. In DOCA/salt mice, relaxation of resistance arterioles was blunted in response to acetylcholine, and to a lesser extent to SNAP, suggesting endothelial dysfunction. Endothelium-dependent vasorelaxation was restored by the potassium-enriched diet.

Conclusion: This study is the first to demonstrate a protective effect of potassium on endothelium-dependent vasorelaxation in the absence of confounding anti-hypertensive effects, as observed in most animal models and the clinical situation. We propose that the known cardio- and nephro-protective effects of potassium might - at least in part - be mediated by the salutary effects on endothelium-dependent arteriolar relaxation.
\end{abstract}

\section{Introduction}

The renal and cardiovascular protector effects of a potassiumenriched diet have been described in large epidemiological studies (Ascherio et al., 1992; Khaw and Barrett-Connor, 1987; Tobian, 1988), as well as in diverse animal models (Ellis et al., 1992; jin et al., 1999; Kido et al., 2008; Wang et al., 2005), which have demonstrated that dietary potassium supplementation prevented vascular and renal injury caused by high salt intake. In this cardiovascular protector context, a pivotal role has been assigned to the anti-hypertensive effects of potassium, as well as to other vasoprotective mechanisms, such as the reduced production of reactive oxygen species (Matsui et al., 2006) and the direct

\footnotetext{
The work reported herein is part of the doctoral dissertation of Caroline Wyss.

* Corresponding author at: Institute of Pathology, Centre Hospitalier Universitaire Vaudois, Rue du Bugnon 25, CH-1011 Lausanne, Switzerland.

E-mail address: hansanton.lehr@yahoo.de (H.A. Lehr).

1 The first two authors have contributed equally to this work.
}

inhibition of intimal hyperplasia (Kido et al., 2008). Several authors have described a beneficial effect of potassium on the generation and release of nitric oxide (NO; see Zhou et al., 1999; Zhou et al., 2000) and a restoration of endothelium-dependent vasorelaxation has been shown in diverse hypertensive rat models (Sudhir et al., 1993; Sugimoto et al., 1988; Zhou et al., 1999). Yet, in these studies, the capacity of potassium supplementation to preserve endothelium-dependent vasorelaxation was always accompanied by a reduction of blood pressure in treated animals, making it impossible to know whether the salutary effect of potassium on vascular function was direct or whether it was indirect, secondary to its antihypertensive action. Ex vivo studies on explanted rings of muscular arteries have not conclusively argued against this latter possibility because of the artificial ex vivo conditions and the study of large caliber conductance vessels that physiologically exert quite different functions than the precapillary resistance arterioles that are operative in blood pressure regulation (Schaefer et al., 2012).

Several years ago, we have described a mouse model, which develops striking cardiac and renal hypertrophy when one renin gene mice are fed 
a DOCA/salt diet, notably in the absence of arterial hypertension (Wang et al., 2002), and in which cardiovascular pathology is completely prevented by dietary potassium supplementation (Wang et al., 2005). In contrast, two-renin gene mice do develop hypertension under the same condition (Wang et al., 2002). We have hence asked the question whether DOCA/salt treated one-renin mice might be suitable to demonstrate a direct effect of potassium diet onendothelium-dependent vasorelaxation, in the absence of confounding anti-hypertensive effects. In order to examine the relaxation of pre-capillary resistance arterioles under conditions of an intact animal organism, we used intravital microscopy in mice chronically instrumented with a dorsal skinfold chamber (Lehr et al., 1993; Schaefer et al., 2005). We show herein that DOCA/ salt treatment attenuates endothelium-dependent vasorelaxation (in the absence of arterial hypertension) and that vasorelaxation is effectively restored in potassium-supplemented mice.

\section{Methods and materials}

The studies described herein comply with appropriate ethical standards. All experiments have been announced to and approved by the local Animal Care and Use Committee prior to the beginning of the experiments. DOCA/salt model: as described previously (Wang et al., 2002), seven week-old male C57BL/6J mice with one renin gene (Ren-1c), weighing 24 to $26 \mathrm{~g}$, were anesthetized with $1 \%$ halothane mixed with oxygen. A silicone tube filled with $35 \mathrm{mg}$ deoxycorticosterone acetate (DOCA, Sigma) with a release rate of about $0.5 \mathrm{mg} /$ day was implanted subcutaneously into the abdominal wall. The tap water was supplemented with $1 \% \mathrm{NaCl}$ (DOCA/salt group). Control mice also underwent uni-nephrectomy without DOCA administration and without the dietary salt supplement (tap water group). All mice received standard maintenance chow, containing $3 \mathrm{mg} \mathrm{Na}^{+}$and $7.5 \mathrm{mg} \mathrm{K}^{+} / \mathrm{g}$ (U.A.R., Villemoisson sur Orge, France) and were housed in a temperature- and humidity-controlled room with an automatic 12:12 h light-dark cycle.

Three weeks before the intravital microscopic experiments, one half of the DOCA/salt treated mice received an additional $0.4 \% \mathrm{KCl}$ in the salt-supplemented drinking water (DOCA/salt + potassium group). The amount of daily water intake was measured and found to be significantly elevated in the two DOCA/salt groups, but with no significant differences between the animals with or without potassium supplementation (Table 1). A total of 9-12 mice were entered into each treatment group. All mice were investigated by intravital microscopy (see below) after 8 weeks of DOCA/salt administration. The tables and figures denote the exact number of animals that were eventually analyzed in each treatment arm.

One day after completion of the intravital microscopic experiments, blood pressure and heart rate were recorded intraarterially using a computerized data-acquisition system as reported previously in detail (Wang et al., 2002). For the placement of the intraarterial catheter, mice were anesthetized via inhalation of $1 \%$ halothane with oxygen. The right carotid artery was exposed in an area of approximately $4 \mathrm{~mm}$ and a PE-10 catheter filled with a heparin $(300 \mathrm{IU} / \mathrm{ml})$ saline solution was inserted into the artery and fixed by silk sutures. The catheter was then subcutaneously tunneled to exit at the back of the neck and fixed with surgical suture material to the titanium observation chambers (see below). Mice were allowed $3 \mathrm{~h}$ to recover from the anesthesia and were then placed in Plexiglas tubes for immobilization. Thirty minutes later, the arterial line was connected to a pressure transducer and blood pressure and heart rate were monitored every $20 \mathrm{~s}$ for $15-30 \mathrm{~min}$.

After blood pressure measurements, $300 \mathrm{ml}$ of blood was retrieved from the carotid artery catheter in a $0.6 \mathrm{ml}$ Multivette tube containing gel/clot activator (Sarstedt, Nümbrecht, Germany). Serum sodium and potassium levels were measured by flame photometry (Instrumentation Laboratory Model 943, Milan, Italy). Pilot studies from our laboratory had shown that this procedure is significantly less stressful for the animals than the other techniques of blood sampling as evidenced by 6 -fold or 2-fold lower plasma norepinephrine levels when compared to decapitation or retroorbital plexus puncture, respectively (Grouzmann et al., 2003). This is particularly important for the measurement of plasma renin concentration and activity, which is notoriously difficult to measure in a way that precludes artifacts due to anesthesia (Iversen and Andersen, 1983) and stressful sampling conditions (Jiménez et al., 1985). Plasma renin activity (PRA) and plasma renin concentration (PRC) were measured as described previously in detail (Wang et al., 2002). After blood pressure measurements and blood sampling, mice were euthanized by an overdose of pentobarbital sodium.

For the intravital microscopic experiments, we used the dorsal skinfold chamber preparation (Lehr et al., 1993). This model permits the microscopic investigation of pre- and postcapillary microvessels and of nutritional capillary perfusion in a fine, striated skin muscle in non-anesthetized animals (Lehr et al., 1993; Schaefer et al., 2005, 2012). After completion of the DOCA/salt treatment, mice with a body weight of 28-32 g were anesthetized with xylazine-ketamine and titanium observation chambers were implanted into the dorsal skinfold as described previously in detail (Lehr et al., 1993). Two days after chamber implantation, the tissue within the observation chambers was examined at scanning magnification $(2 \times$-fold magnification, using a Leica ES5 stereo microscope, with $10 \times$ eyepieces, total magnification 20 -fold) for signs of inflammation (increased microvessel tortuosity and reduced blood cell velocity), edema (incapacity to focus on microvessel edges) or flow obstruction (venous or venular dilation). Since we strictly applied these criteria for what can be considered a chamber with physiological flow conditions, we eliminated $40 \%$ of the animals before the intravital microscopic studies. This number compares well to experiences made in other immunocompetent mouse species (C57BL/6, BALB/c, and ApoE KO, all showing an elimination rate of around 40\%, see Li and Förstermann, 2000; Schaefer et al., 2005), but was slightly higher when compared to mice that are genetically hairless (nu/nu or nude, showing an elimination rate of around 25\%; see Lehr et al., 1993). It should be acknowledged that the success rate of the chamber preparation depends largely on the quality of microsurgical technique and hence varies from experimentor to experimentor (unpublished observation), and that unexpected results are obtained in experiments in which the above described strict criteria are neglected (Schäfer et al., 2005). To eliminate the effects of anesthesia and direct surgical trauma on the microvasculature, a recovery period of 72-96 h was allowed between the implantation of the observation chambers and the intravital microscopic investigations. For intravital microscopy, awake mice were placed in a Plexiglas tube fixed to a Plexiglas platform under a diagnostic microscope (Leica Dialux-20, Fig. 1) and the superfusion protocol performed as explained in detail in previous publications (Schaefer et al., 2005, 2012). For the intravital microscopic

Table 1

Mean arterial blood pressure (MAP), heart rate (HR), serum sodium and potassium levels, plasma renin activity (PRA), plasma renin concentration (PRC) and water intake in DOCA/ salt-treated one-renin gene mice. Data are mean \pm SEM of $10-12$ animals per group, DOCA/salt versus control and versus DOCA $/$ salt + potassium. ${ }^{*} \mathrm{P}<0.05$, ${ }^{* *} \mathrm{P}<0.01$, ${ }^{* * *} \mathrm{P}<0.001$, one-way ANOVA analysis followed by a Newman-Keuls test.

\begin{tabular}{|c|c|c|c|c|c|c|c|}
\hline & MAP (mm Hg) & HR (bpm) & Serum $\mathrm{Na}^{+}(\mathrm{mmol} / \mathrm{l})$ & Serum $\mathrm{K}^{+}(\mathrm{mmol} / \mathrm{l})$ & PRA (ng/ml/h) & PRC (ng/ml/h) & Water intake ( $\mathrm{ml} / \mathrm{day} / 25 \mathrm{~g}$ body $\mathrm{wt})$ \\
\hline \multicolumn{8}{|l|}{ Group } \\
\hline Tap water $(n=10)$ & $116 \pm 2$ & $606 \pm 14$ & $152 \pm 1$ & $4.6 \pm 0.1$ & $5.5 \pm 1.3$ & $387 \pm 87$ & $6.3 \pm 0.3$ \\
\hline DOCA $/$ salt $(n=9)$ & $113 \pm 2$ & $586 \pm 09$ & $153 \pm 1$ & $3.1 \pm 0.1^{* *}$ & $0.9 \pm 0.6^{*}$ & $35 \pm 25^{* * *}$ & $27.4 \pm 1.3^{* * *}$ \\
\hline DOCA/salt + K $(n=9)$ & $112 \pm 3$ & $584 \pm 10$ & $154 \pm 1$ & $4.4 \pm 0.1$ & $2.1 \pm 1.2^{*}$ & $91 \pm 44^{* *}$ & $31.1 \pm 1.2^{* * *}$ \\
\hline
\end{tabular}


studies, two or three arteriolar segments (with diameters ranging from 20 to $60 \mathrm{~mm}$ ) were chosen either from the immediate proximity of bifurcations (to facilitate repeated identification of the same vessel segment) or at a distance of more than $300 \mu \mathrm{m}$ from bifurcations. After an equilibration period of $30 \mathrm{~min}$ following the start of the tissue superfusion, the arteriolar segments were observed at $100 \times$ magnification $(10 \times$ infinity plan achromatic objective, $10 \times / 0.25$, with subsequent 10-fold numeric magnification) for $1 \mathrm{~min}$ intervals and the video images were recorded on videotape using a 1-chip, color, CCD camera (TK-C1381; JVC) and a standard hard disk video recorder (NV-HS900; Panasonic). Using this technical setup, a minimal detectable size difference of the microvascular segments of $1 \mu \mathrm{m}$ can be reliably identified. The same arteriolar vessel segments - thoroughly documented on microvascular maps that were generated for each animal from videotaped low-magnification chamber overviews - were observed again after a 5 min superfusion period with acetylcholine in increasing concentrations $(0.1-10 \mathrm{mM})$ and, following a 10 min recovery period, after a 5-min superfusion period with S-nitroso-N-acetyl-D,L-penicillamine (SNAP, $10 \mathrm{mM}$ ).

For quantification of the intravital microscopic findings, video still images were imported at a resolution of 72 dpi into Photoshop (Adobe, San Jose, CA, version CS2) run on a Macintosh G4 computer (Apple computers, Cupertino, CA). Assessment of microvessel diameters was performed using Photoshop-based image analysis as descried previously (Brunner et al., 2000). A total of five measurements were taken per vessel segment and time point, and mean values calculated in order to eliminate putative outlier data. All data in the figures are rendered as mean \pm SD. In analogy to previous publications (Schaefer et al., 2005, 2012), endothelium-dependent (i.e. acetylcholine-induced) vasorelaxation was calculated as percent of total endothelium-independent (i.e. SNAPinduced) vasorelaxation (indicated as ACh/SNAP in Fig. 3). Statistical differences between mean values of the various treatment groups were determined using the ANOVA test with post-hoc Newman-Keuls correction (GraphPad Software, version 5.0, La Jolla, CA).

\section{Results}

In analogy to previous observations (Wang et al., 2005), DOCA/salt treatment for eight weeks resulted in significant hypokalemia in uninephrectomized one renin gene mice (Table 1 ). At the same time, serum sodium was not changed by DOCA/salt treatment (Table 1 ). As expected, no effects of DOCA/salt treatment were observed on mean arterial blood pressure and on heart rate (Table 1). Potassium supplementation for three weeks restored serum potassium in DOCA/salt mice to levels of control mice and did not affect any of the macrohemodynamic parameters (Table 1 ).

Acetylcholine superfusion of the chamber tissue under intravital microscopic observation showed a dose-dependent vasorelaxation of precapillary resistance arterioles in control mice (black bars in Fig. 2). From pilot studies during the establishment of the superfusion model, we have learnt that the highest acetylcholine dose we used in our present study causes the maximum of acetylcholine-dependent vasodilation in control animals, corresponding to roughly $80 \%$ of the maximum potential of vasodilation of the vessel segment, induced by a mix of papaverine, bradykinin and SNAP. We now found that acetylcholine-induced vasorelaxation was significantly inhibited in the DOCA/salt-treated one renin gene mice (empty bars in Fig. 2). Note that over the entire dose range of acetylcholine superfusion, the maximal response was less than half of the response observed in control mice. Also, the vasorelaxing response to the NO donor SNAP was blunted by DOCA/salt, albeit much less so than the response to acetylcholine (Fig. 2). When the relaxing responses to SNAP and acetylcholine $(10 \mu \mathrm{M})$ were calculated as a percent ratio for each mouse, this ratio was reduced by more than $50 \%$ in DOCA/ salt mice (Fig. 3), connoting a significant attenuation of endotheliumdependent vasorelaxation. Feeding DOCA/salt-treated mice for three weeks prior to the intravital microscopic experiments with potassium restored endothelium-dependent and -independent vasorelaxation (gray bars in Fig. 2) and the percent ratio of acetylcholine over SNAPinduced vasorelaxation (gray bar in Fig. 3).

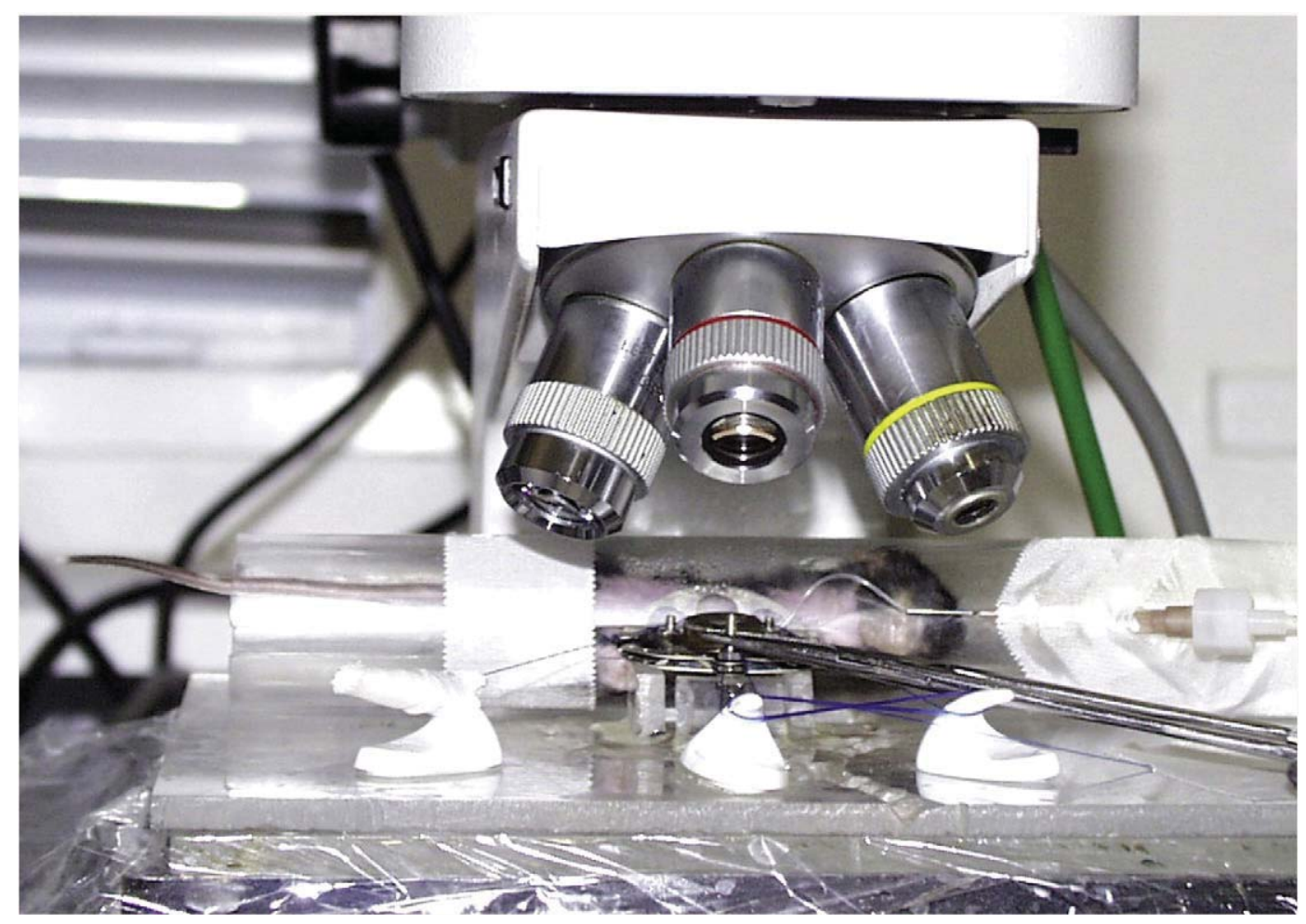

Fig. 1. Dorsal skinfold chamber model in mice and microscope setup. C57BL mice were chronically instrumented with titanium skinfold chamber in the dorsal skinfold. For intravital microscopy, the mice were positioned in a Plexiglas tube that was placed sideways under the microscope. An intraarterial polyethylene catheter was attached to a pressure transducer (right). All intravital microscopic examinations of the microcirculation in the observation chambers were performed in awake mice, thus eliminating any putative adverse effects of anesthetic agents. 


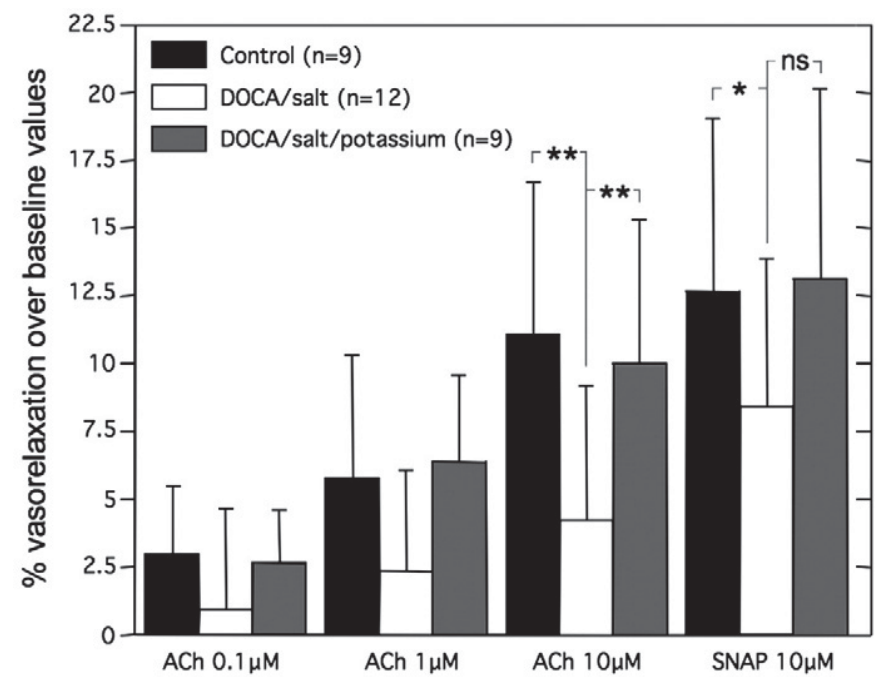

Fig. 2. Increase of diameters of precapillary resistance arterioles in response to superfusion with various doses of acetylcholine (ACh, $0.1-10 \mu \mathrm{M})$ or SNAP $(10 \mu \mathrm{M})$. Data are shown as percent increase over baseline values. Data are mean \pm SD of 9-12 animals per group. Black bars indicate control mice, white bars DOCA/salt treated mice, and gray bars DOCA/salt + potassium treated mice. Note that potassium normalizes the endothelium-dependent as well as the endothelium-independent vasorelaxation in DOCA/salt mice to levels of untreated control mice. Asterisks indicate statistically significant differences at the ${ }^{*} \mathrm{P}<0.05$ and ${ }^{* *} \mathrm{P}<0.01$ level.

Please note that the number of animals in the respective macrohemodynamic and intravital microscopic measurements is not always identical, because some animals were lost after the intravital microscopy measurements $(n=12$ intravital microscopic versus $n=9$ macrohemodynamic measurements in the DOCA/salt group) or because the intravital microscopy measurements could not be reliably analyzed due to edema formation in the chamber $(n=9$ intravital microscopic versus $n=10$ macrohemodynamic measurements in the control group).

\section{Discussion}

The principal finding of our study is the observation that endothelium-dependent and - to a lesser extent - endotheliumindependent vasorelaxation are preserved by a potassium diet in DOCA/salt mice (Figs. 2, 3), independent of confounding antihypertensive effects (Table 1) This finding is novel in that none of the prior experimental approaches used by various groups in the past have been able to separate putative antihypertensive effects of potassium from its direct effects on endothelium-dependent vasorelaxation. In that respect, we believe that our study confirms, and in a way complements the observations by Wu and coworkers, who showed that feeding DOCA/salt treated rats potassium in the form of a diet rich in milk minerals restored acetylcholine-induced relaxation in aortic rings ex vivo (Wu et al., 1996). The combined use of DOCA/salt mice and intravital microscopy has now allowed to eliminate not only putative macrohemodynamic effects (Table 1 ), but also potentially confounding effects of anesthesia. This is particularly relevant because anesthetic agents have previously been found to impact on nitric oxide independent acetylcholine-induced endothelial vasorelaxation (de Wit et al., 1999).

As shown in Table 1, the potassium serum levels in the DOCA/ salt + potassium treated animals were well in the range of the potassium levels measured in control animals. In fact, the small difference of potassium levels in the different groups is unlikely to have a direct operative effect on smooth muscle relaxation, such as hyperpolarization via opening of $\mathrm{KCa}^{++}$-channels. Furthermore, opening/rectifying inward $\mathrm{K}^{+}$channels or a transient stimulation of the electrogenic $\mathrm{Na} / \mathrm{K}$ pump, that can be seen in smaller immediate elevations of extracellular

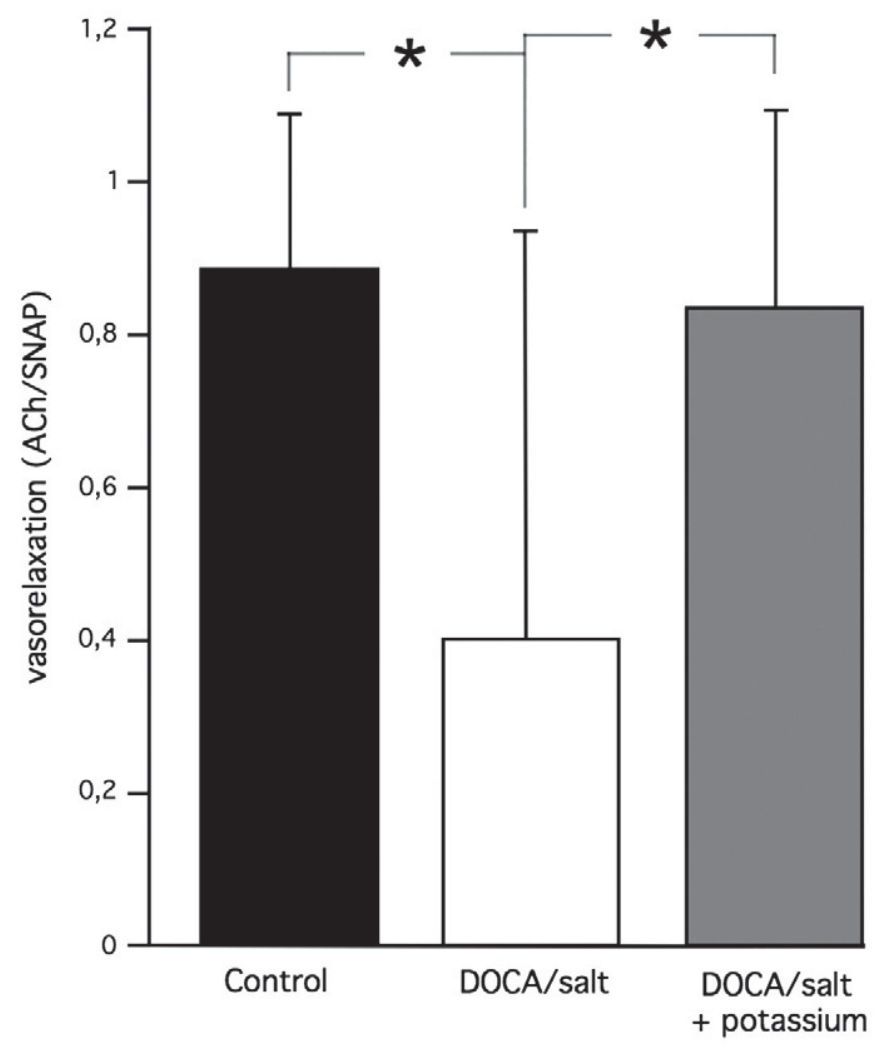

Fig. 3. Normalization of endothelium-dependent and endothelium-independent vasorelaxation in DOCA/salt treated mice by potassium administration. Shown in this graph is the ratio of acetylcholine $(\mathrm{ACh})$ over SNAP-induced vasorelaxation at doses of $10 \mu \mathrm{M}$ (both agents). Note that DOCA/salt significantly suppresses endothelium-dependent vasorelaxation, as evidenced by a marked drop of the Ach/SNAP ratio, and that this effect is effectively counteracted by the potassium diet to levels of control animals. Data are mean \pm SD of $n=9$ control mice (black bars), $n=12$ DOCA/salt mice (white bars) and $\mathrm{n}=9$ DOCA/salt mice with the potassium diet (gray bars). The asterisk denotes a statistically significant difference between data in DOCA/salt mice and control mice and between data in DOCA/salt mice with or without the potassium diet at the ${ }^{*} \mathrm{P}<0.05$ level.

$\mathrm{K}^{+}$, is likely not operative in our model. Therefore, we propose that potassium supplementation might restore vasorelaxation through as yet unknown indirect effects, which might include reduction of vascular oxidative stress (Matsui et al., 2006) or other mechanisms. Endothelial nitric oxide release is dependent on various cofactors such as tetrahydrobiopterin (BH4), which is important to keep the eNOS in a coupled state. DOCA/salt treatment decreases BH4 levels in hypertensive mice which results in eNOS uncoupling and increased $\mathrm{O}_{2}^{-}$production (Du et al., 2008; Xie et al., 2010). The reduction of BH4 in DOCA/salt hypertensive mice has been discussed to be due to a reduced de novo synthesis and/or increased oxidation to BH3. Administration of $\mathrm{BH} 4$ in combination with the NADPH inhibitor apocynin has been shown to increase the bioavailability of NO via eNOS recoupling in aortic rings of DOCA/salt hypertensive mice ex vivo (Youn et al., 2012). These results provide a possible explanation why vasorelaxation in response to acetylcholine was attenuated in the DOCA/salt treated mice in our study.

One of the key attributes of the present study is the fact that we used intravital microscopy (Fig. 1) to study precapillary resistance arterioles, which are relevant for blood pressure regulation, and not conductance vessels, which are frequently examined ex vivo. These large-caliber vessels, such as aortic or carotid rings, differ not only in terms of anatomical structure, but also in terms of functionality, including the endothelial control over vascular tone (Mulvany and Aalkjaer, 1990). Based on the findings in mouse coronary vessels, Guillot et al. (1999) identified vascular bed-specific pathways of eNOS regulation with striking differences in eNOS gene expression between microvessels (strong uniform expression) and larger arteries (patchy expression with accentuation around 
branching sites). Parent and coworkers found that inhibiting NO synthesis selectively reduced acetylcholine-induced vasodilatation in cardiac resistance vessels of dogs, but failed to prevent similar responses in conductance coronary vessels (Parent et al., 1996). Recently, our group has shown, using the same mouse model as applied in the present study, that angiotensin-II-mediated hypertension (2 kidney/1 clip Goldblatt model) leads to endothelium-dependent vasodysfunction in precapillary resistance arterioles, while this effect could not be reproduced in rings of large caliber conductance vessels from the same animals ex vivo (Schaefer et al., 2012).

Can the observations made in the mice of our study be translated into a hypertensive organism? Tobian and colleagues have shown that stroke-prone SHR and Dahl salt sensitive hypertensive rats fed a high potassium diet had a significant reduction in death rate even though blood pressure was similar in the low and high potassium diet groups (Sugimoto et al., 1988; Tobian et al., 1985). The precise mechanism by which dietary potassium reduces death has yet to be demonstrated. One could speculate that dietary potassium might protect the integrity of endothelial cells even when they are under heightened stress from the elevated blood pressure, thus preventing artery wall lesions with subsequent cerebral hemorrhage and infarcts. We have demonstrated previously that DOCA/salt induces cardiac and renal hypertrophy, hypokalemia and metabolic alkalosis independent of blood pressure in 1-renin gene mice. Correction of hypokalemia and metabolic alkalosis by dietary potassium supplementation can prevent the development of renal and cardiac hypertrophy, as well as vascular remodeling (unpublished data) in normotensive, 1-renin gene mice that receive excess mineralocorticoid and salt (Wang et al., 2002, 2005). Nevertheless, the exact mechanisms of renal and cardiovascular protection by dietary potassium in human hypertension are currently still unclear and subject of ongoing clinical trials (effects of potassium salts on blood pressure and target organ damage and potassium intake in patients with chronic kidney disease; http://clinicaltrials.gov/ct2/show/NCT00949585). Finally, we should note that with the choice of the particular animal model in our study we wanted to specifically resolve an experimental dilemma (antihypertensive versus direct pharmacological effects of a potassiumenriched diet on endothelium-dependent vasorelaxation in resistance vessels), and not present an exact animal model of a specific human disease.

In conclusion, the present experiments confirm a salutary role of dietary potassium on endothelium-dependent vasorelaxation. We speculate that the cardioprotection by potassium might be mediated by putative antioxidant effects (Matsui et al., 2006), ameliorating the availability/ efficacy of $\mathrm{NO}$ and its well-established cardioprotector actions ( $\mathrm{Li}$ and Förstermann, 2000)

\section{Acknowledgments}

The excellent technical assistance of Jean-Christophe Wyss is gratefully acknowledged.

The authors declare that there is no conflict of interest.

\section{References}

Ascherio, A., Rimm, E.B., Giovannucci, E.L., Colditz, G.A., Rosner, B., Willett, W.C., Sacks, F., Stampfer, M.J., 1992. A prospective study of nutritional factors and hypertension among US men. Circulation 86, 1475-1484.

Brunner, J., Krummenauer, F., Lehr, H.A., 2000. Quantification of video-taped images in microcirculation research using inexpensive imaging software (Adobe Photoshop). Microcirculation 7, 103-107. de Wit, C., Esser, N., Lehr, H.A., Bolz, S.S., Pohl, U., 1999. Pentobarbital-sensitive EDHF comediates ACh-induced arteriolar dilation in the hamster microcirculation. Am. J. Physiol. 276, H1527-H1534.

Du, Y.H., Guan, Y.Y., Alp, N.J., Channon, K.M., Chen, A.F., 2008. Endothelium-specific GTP cyclohydrolase I overexpression attenuates blood pressure progression in saltsensitive low-renin hypertension. Circulation 117, 1045-1054.

Ellis, D., Banner, B., Janosky, J.E., Feig, P.U., 1992. Potassium supplementation attenuates experimental hypertensive renal injury. J. Am. Soc. Nephrol. 2, 1529-1537.

Grouzmann, E., Cavadas, C., Grand, D., Moratel, M., Aubert, J.F., Brunner, H.R., Mazzolai, L., 2003. Blood sampling methodology is crucial for precise measurement of plasma catecholamines concentrations in mice. Pflugers Arch. 447, 254-258.

Guillot, P.V., Guan, J., Liu, L., Kuivenhoven, J.A., Rosenberg, R.D., Sessa, W.C., Aird, W.C., 1999. A vascular bed-specific pathway regulates cardiac expression of endothelial nitric oxide synthase. J. Clin. Invest. 103, 799-805.

Iversen, B.M., Andersen, K.J., 1983. The effect of sampling conditions on rat plasma renin. Comp. Biochem. Physiol. A Comp. Physiol. 74, 331-332.

Jiménez, W., Martínez-Pardo, A., Arroyo, V., López, C., Rímola, A., Gaya, J., Rivera, F., 1985 Effect of the method of blood extraction on plasma levels of renin in the Wistar rat Rev. Esp. Fisiol. 41, 299-303.

Jin, L., Chao, L., Chao, J., 1999. Potassium supplement upregulates the expression of renal kallikrein and bradykinin B2 receptor in SHR. Am. J. Physiol. 276, F476-F484.

Khaw, K.T., Barrett-Connor, E., 1987. Dietary potassium and stroke-associated mortality: a 12 year prospective population study. N. Engl. J. Med. 316, 235-240.

Kido, M., Ando, K., Onozato, M., Tojo, A., Yoshikawa, M., Ogita, T., Fujita, T., 2008. Protective effect of dietary potassium against vascular injury in salt-sensitive hypertension. Hypertension 51, 225-231.

Lehr, H.A., Leunig, M., Menger, M.D., Nolte, D., Messmer, K., 1993. Dorsal skinfold chambe technique for intravital microscopy in nude mice. Am. J. Pathol. 143, 1055-1062.

Li, H., Förstermann, U., 2000. Nitric oxide in the pathogenesis of vascular disease. J. Pathol. 190, 244-254.

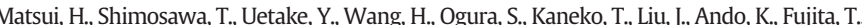
2006. Protective effect of potassium against the hypertensive cardiac dysfunction. Association with reactive oxygen species reduction. Hypertension 48, 225-231.

Mulvany, M.J., Aalkjaer, C., 1990. Structure and function of small arteries. Physiol. Rev. 70, 921-961.

Parent, R., Hamdad, N., Ming, Z., Lavallee, M., 1996. Contrasting effects of blockade of nitric oxide formation on resistance and conductance coronary vessels in conscious dogs. Cardiovasc. Res. 31, 555-567.

Schaefer, S.C., Wallerath, T., Closs, E.I., Schmidt, C., Schwarz, P.M., Förstermann, U., Lehr, H.A. 2005. Dexamethasone suppresses eNOS and CAT-1 and induces oxidative stress in mouse resistance arterioles. Am. J. Physiol. 288, H436-H444.

Schaefer, S.C., Pellegrin, M., Wyss, C., Aubert, J.F., Nussberger, J., Hayoz, D., Lehr, H.A. Mazzolai, L., 2012. Intravital microscopy reveals endothelial dysfunction in resistance arterioles in angiotensin II-induced hypertension. Hypertens. Res. 35, 855-861.

Schäfer, S.C., Sehrt, D.M., Kamler, M., Jakob, H., Lehr, H.A., 2005. Paradoxical attenuation of leukocyte rolling in response to ischemia-reperfusion and extracorporeal blood circulation in inflamed tissue. Am. J. Physiol. Heart Circ. Physiol. 289, H330-H335.

Sudhir, K., Kurtz, T.W., Yock, P.G., Conolly, A.J., Morris Jr., R.C., 1993. Potassium preserves endothelial function and enhances aortic compliance in Dahl rats. Hypertension 22 315-322.

Sugimoto, T., Tobian, L., Ganguli, M.C., 1988. High potassium diets protect against dysfunction of endothelial cells in stroke-prone spontaneous hypertensive rats. Hypertension $11,579-585$

Tobian, L, 1988. Potassium and hypertension. Nutr. Rev, 46, 273-283.

Tobian, L., Lange, J., Ulm, K., Wold, L., Iwai, J., 1985. Potassium reduces cerebral hemorrhage and death rate in hypertensive rats, even when blood pressure is not lowered. Hypertension 7, I110-I114.

Wang Q Hummeler, E, Nussberger, J. Clement, S, Gabbiani, G, Brunner, H.R, Burnier, M 2002. Blood pressure, cardiac, and renal responses to salt and deoxycorticosterone acetate in mice: role of renin genes. J. Am. Soc. Nephrol. 13, 1509-1516.

Wang, Q., Domenighetti, A.A., Pedrazzini, T., Burnier, M., 2005. Potassium supplementation reduces cardiac and renal hypertrophy independently of blood pressure in DOCA/salt mice. Hypertension 46, 547-554

Wu, X., Mäkynen, H., Korpela, R., Pörsti, I., 1996. Whey mineral supplementation and arterial tone in mineralocorticoid- $\mathrm{NaCl}$ hypertension. Cardiovasc. Res. 32, $1115-1122$.

Xie, H.H., Zhou, S., Chen, D.D., Channon, K.M., Su, D.F., Chen, A.F. 2010. GTP cyclohydrolase I/ BH4 pathway protects EPCs via suppressing oxidative stress and thrombospondin-1 in salt-sensitive hypertension. Hypertension 56, 1137-1144.

Youn, J.Y., Wang, T., Blair, J., Laude, K.M., Oak, J.H., McCann, L.A., Harrison, D.G., Cai, H. 2012. Endothelium-specific sepiapterin reductase deficiency in DOCA-salt hypertension. Am. J. Physiol. Heart Circ. Physiol. 302, H2243-H2249.

Zhou, M.S., Nishida, Y, Yoneyama, H., Chen, Q.H., Kosaka, H., 1999. Potassium supplementation increases sodium excretion and nitric oxide production in hypertensive Dahl rats. Clin. Exp. Hypertens. 21, 1397-1411.

Zhou, M.S., Kosaka, H., Yoneyama, H., 2000. Potassium augments vascular relaxation mediated by nitric oxide in the carotid arteries of hypertensive Dahl rats. Am. J. Hypertens. 13, 666-672. 